Supporting Information for article:

\title{
How well can you tailor the charge of lipid vesicles?
}

\author{
Gilbile, D., Docto D., Kingi D.T., Kurniawan, J., Monahan D., Tang A., and Kuhl, T.L.* \\ Department of Chemical Engineering, University of California at Davis, CA, USA.
}

Corresponding Author
*E-mail: tlkuhl@ucdavis.edu.

\section{Table of Contents:}

Section S1: Lipid monolayer surface pressure vs. area (П-A) isotherms.

Section S2: Fluorescence microscopy of supported lipid bilayers.

Section S3: Vesicle size measurements (DLS)

Section S4: SFA force/radius vs. distance profiles for all lipid bilayer compositions.

Section S5: Plane of origin of charge analysis for SFA experiments. 


\section{Section S1: Lipid monolayer surface pressure-area (П-A) isotherms.}
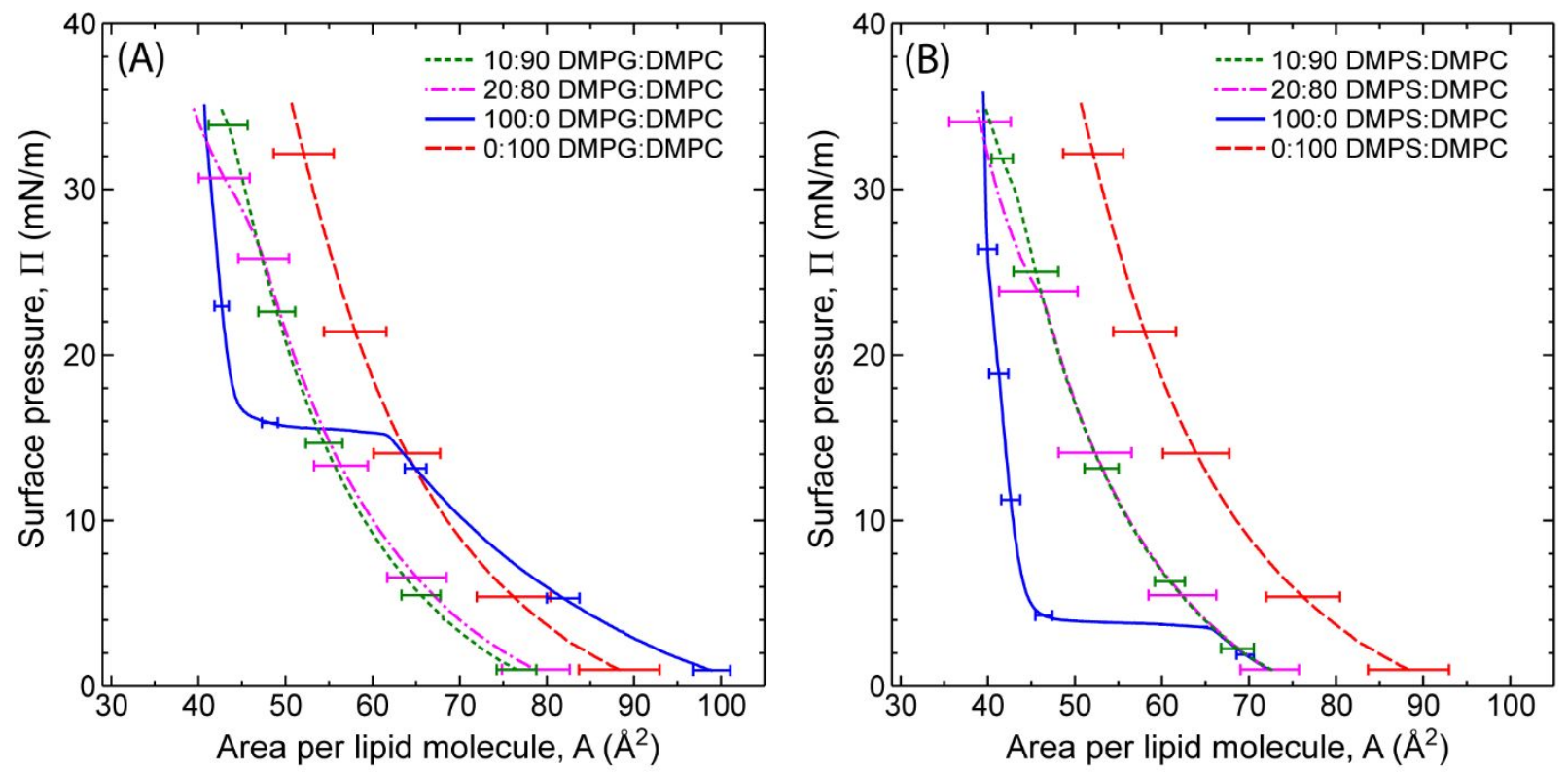

Figure S1: Pressure vs. area ( $\Pi-A)$ isotherm curves for different compositions of (A) DMPG:DMPC and (B) DMPS:DMPC monolayers at the air-water interface, at room temperature $\left(24.5 \pm 1.0^{\circ} \mathrm{C}\right)$ on subphase of milliQ water. Selected error bars shown are \pm 1 standard deviation.

Lipid monolayers were prepared by spreading the desired lipid solution at the air-water interface on a Langmuir Blodgett Trough. The solvent was allowed to evaporate for 10 minutes, after which the lipids were compressed at a constant rate of $20 \mathrm{~cm}^{2} / \mathrm{min}$. To check reproducibility, experiments were performed with three independent mixtures made from separate stock solutions of lipids. The isotherms shown represent one set of experimental data with selected error bars. Figure S1 shows the $\Pi-\mathrm{A}$ isotherms for lipid monolayers composed of different lipid mixtures - 10:90, 20:80, 100:0 DMPG:DMPC or DMPS:DMPC at the air-water interface. The isotherms indicate that while pure (single component) DMPC is in the fluid phase, pure DMPS and pure DMPG monolayers are in the gel phase at $35 \mathrm{mN} / \mathrm{m}$. For mixed lipid compositions, the curves indicate that the monolayers are in the fluid phase - gel phase coexistence regime. Upon transfer to a DPPE monolayer-coated mica substrate, these mixed lipid monolayers (outer leaflet) are expected to be in the gel phase. 
The average molecular area for the compositions used in SFA experiments is between $42-45 \AA^{2}$ per lipid (at $35 \mathrm{mN} / \mathrm{m})$.
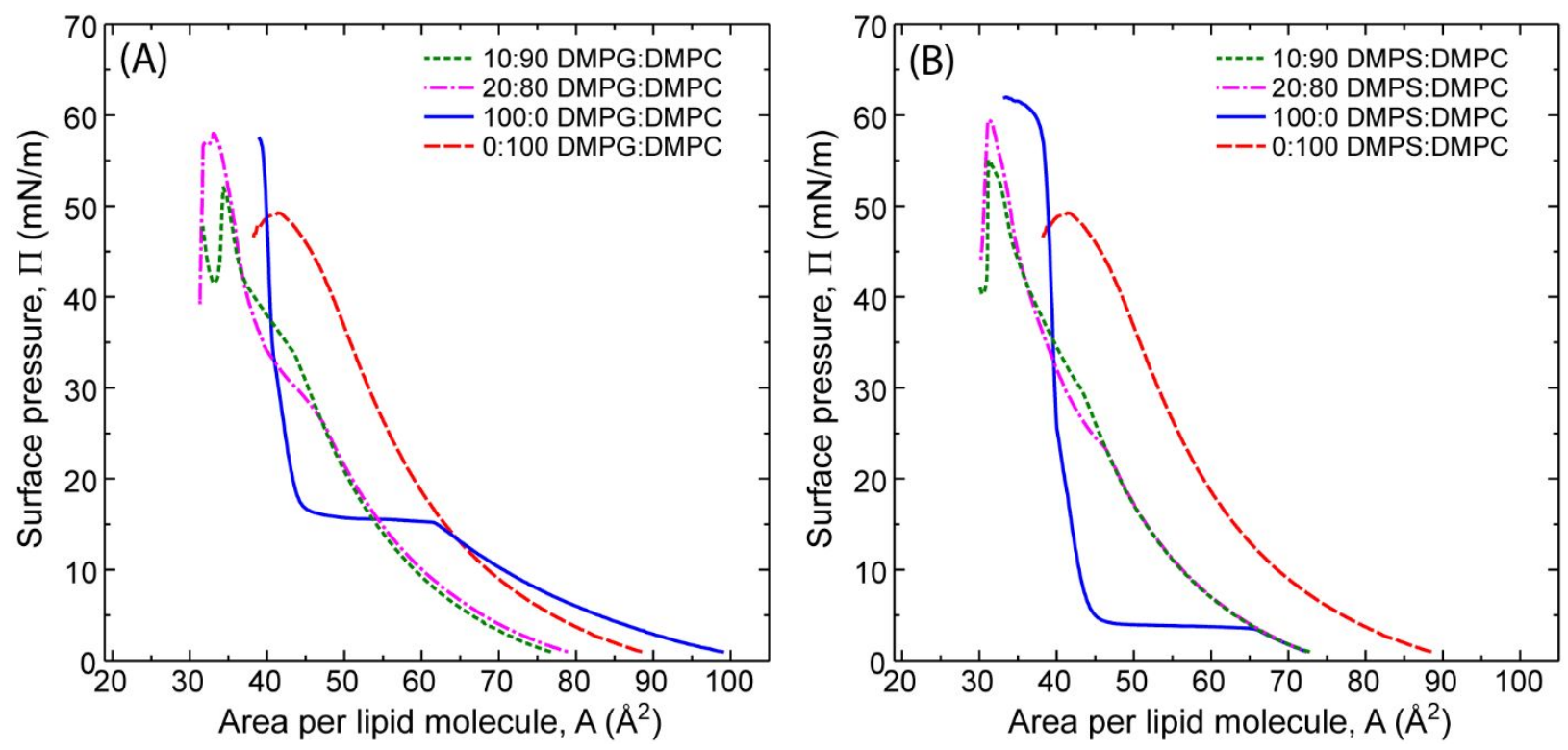

Figure S2: Pressure vs. area ( $\Pi-A)$ isotherm curves to collapse for difference compositions of mixed (A) DMPG: DMPC and (B) DMPS: DMPC monolayers at the air-water interface, at room temperature $\left(24.5 \pm 1.0^{\circ} \mathrm{C}\right)$ on a subphase of milliQ water.

Figure S2 shows the $\Pi-\mathrm{A}$ isotherms to collapse for lipid monolayers composed of different lipid mixtures 10:90, 20:80, 100:0 DMPG:DMPC or DMPS:DMPC at the air water interface. The collapse pressure of pure (single component) lipid monolayers was found to be around $48 \pm 2$ $\mathrm{mN} / \mathrm{m}$ for DMPC, $60 \pm 3 \mathrm{mN} / \mathrm{m}$ for DMPS, and $58 \pm 3 \mathrm{mN} / \mathrm{m}$ for DMPG. For the lipid mixtures containing 10 or $20 \mathrm{~mol} \%$ DMPS/DMPG, the curves indicate a single collapse peak with collapse pressures between those for pure DMPC and pure DMPS/DMPG indicating that the lipids were well mixed. However, phase separation was observed by fluorescence microscopy as shown in figure S3. 
Section S3: Vesicle size measurements (DLS)

Table S1: Size measurements of vesicles in $140 \mathrm{mM}$ phosphate buffer, $\mathrm{pH}$ 7.4.

\begin{tabular}{|c|c|}
\hline DMPS:DMPC & diameter (nm) \\
\hline $0: 100$ & $171 \pm 80$ \\
$0.1: 99.9$ & $137 \pm 26$ \\
$0.5: 99.5$ & $158 \pm 51$ \\
$1: 99$ & $194 \pm 27$ \\
$5: 95$ & $165 \pm 34$ \\
$10: 90$ & $150 \pm 31$ \\
$20: 80$ & $148 \pm 32$ \\
$30: 70$ & $141 \pm 24$ \\
$100: 0$ & $152 \pm 66$ \\
\hline DMPG:DMPC & diameter (nm) \\
\hline $0.1: 99.9$ & $172 \pm 47$ \\
$0.5: 99.5$ & $169 \pm 32$ \\
$1: 99$ & $149 \pm 26$ \\
$5: 95$ & $152 \pm 29$ \\
$10: 90$ & $166 \pm 48$ \\
$20: 80$ & $146 \pm 35$ \\
$30: 70$ & $144 \pm 37$ \\
$50: 50$ & $150 \pm 36$ \\
$100: 0$ & $128 \pm 39$ \\
\hline
\end{tabular}

Table S2: Size measurements of vesicles in $0.5 \mathrm{mM}$ NaNO3, 5.7.

\begin{tabular}{|c|c|}
\hline DMPS:DMPC & diameter $(\mathbf{n m})$ \\
\hline $0.5: 99.5$ & $104 \pm 68$ \\
$1: 99$ & $106 \pm 67$ \\
$10: 90$ & $97 \pm 57$ \\
$20: 80$ & $160 \pm 51$ \\
$100: 0$ & $151 \pm 50$ \\
\hline DMPG:DMPC & diameter $(\mathbf{n m})$ \\
\hline $0.5: 99.5$ & $161 \pm 97$ \\
$2: 98$ & $173 \pm 64$ \\
$10: 90$ & $134 \pm 61$ \\
$100: 0$ & $182 \pm 71$ \\
\hline
\end{tabular}




\section{Section S2: Fluorescence Microscopy of supported lipid bilayers.}

Fluorescence microscopy images were acquired using a Nikon Eclipse E600 optical microscope connected to an Andor Zyla sCMOS camera using a 40× magnification water-immersion objective lens. 5 mol\% fluorescent dye-labelled Texas Red-DHPE lipid was incorporated into lipid bilayers of different compositions used in this work to help visualize the membrane.
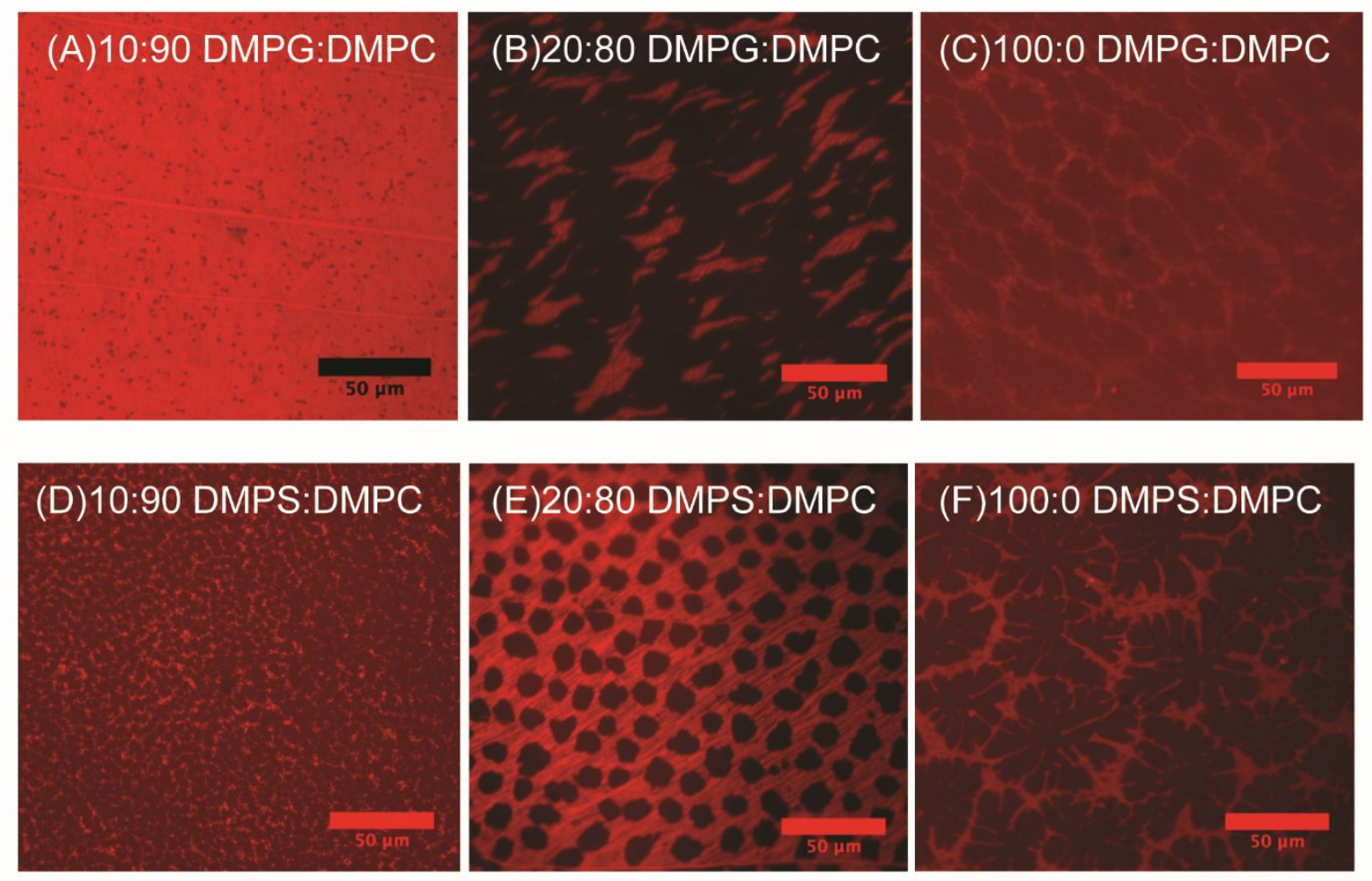

Figure S3: Fluorescence microscopy images of mixed DMPG: DMPC (A-C) and DMPS: DMPC (D-F) supported lipid bilayers on mica. The bilayers contain $5 \mathrm{~mol} \%$ fluorescently labelled Texas Red-DHPE lipid. Scale bars are $50 \mu \mathrm{m}$.

Figure S3 shows fluorescence microscopy images of various compositions of lipid bilayers deposited onto freshly-cleaved mica using the Langmuir Blodgett technique. The inner leaflet of the lipid bilayers was DPPE deposited on mica at $45 \mathrm{mN} / \mathrm{m}$, the outer leaflet (compositions indicated in figure labels, containing $5 \mathrm{~mol} \%$ fluorescently labelled Texas Red-DHPE) was deposited onto DPPE-coated mica at $35 \mathrm{mN} / \mathrm{m}$. The deposition speed was $1 \mathrm{~mm} / \mathrm{min}$ for both inner 
and outer leaflets. Texas Red-DHPE is known to partition in to the fluid phase due to the bulky fluorescent-label bearing lipid head group. ${ }^{1}$ Bilayers composed of mixed DMPG:DMPC or DMPS: DMPC outer leaflets shown in figure S3 (A, B, D, E), show both fluorescent and dark regimes in varying proportion suggesting that there was fluid phase and gel phase coexistence. Subsequent fluorescence recovery after photobleaching (FRAP) experiments on these bilayers (not shown) did not show fluorescence recovery, suggesting that these bilayers behave largely like gel-phase systems. From the $\Pi-A$ isotherms it is clear that pure DMPG and DMPS bilayers are expected to be in the gel phase. This is confirmed by figure $\mathrm{S} 3(\mathrm{C}, \mathrm{F})$ in which we observe large $50-70 \mu \mathrm{m}$ sized dark patches from which the fluorescently labelled lipid is being excluded to the edges due to steric effects. 


\section{Section S4: SFA force/radius vs. distance profiles for all lipid bilayer compositions.}

Figure S4 shows the interaction force profiles for all lipid bilayer compositions (10:90, 20:80, 100:0 DMPG:DMPC or DMPS:DMPC) measured by SFA. Table S1 summarizes the values of surface charge, potential, effective area per dissociated charged lipid, and percentage ionization. Error is one standard deviation based on three independent SFA measurements for each composition.
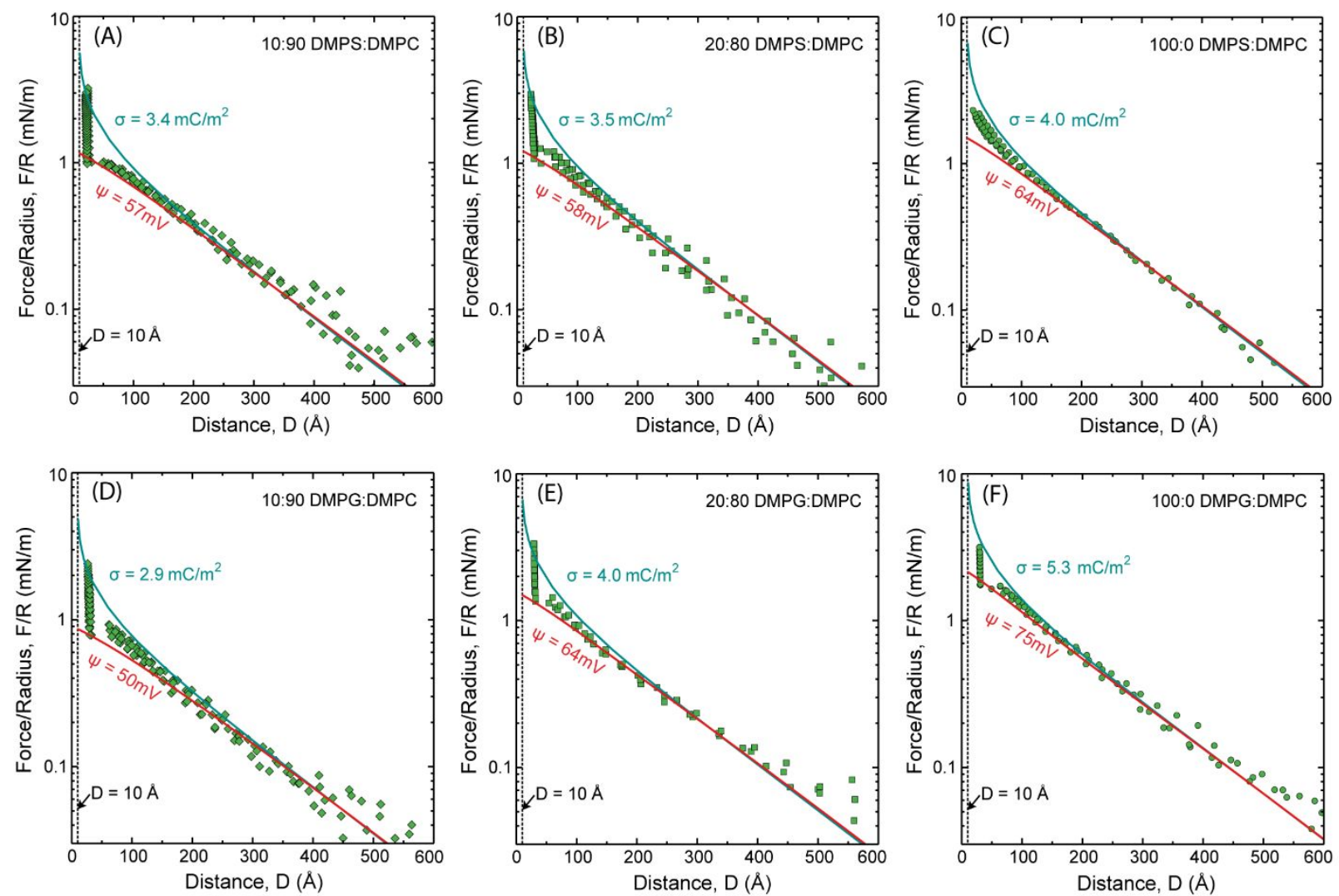

Figure S4: Measured force profiles between lipid bilayers composed of 10:90, 20:80, 100:0 DMPG:DMPC or DMPS:DMPC (outer leaflet) deposited on DPPE (inner leaflet) in $0.5 \mathrm{mM}$ $\mathrm{NaNO}_{3}, \mathrm{pH} 5.7, \mathrm{~T}=25.0^{\circ} \mathrm{C}$ ) on a semi-logarithmic plot. $\mathrm{D}=0 \AA$ was defined as anhydrous bilayer contact which corresponds to the location of the phosphate head group plane. The plane of origin of charge was set at $\mathrm{D}=10 \AA$. 


\section{Section S5: Plane of origin of charge analysis for SFA experiments.}
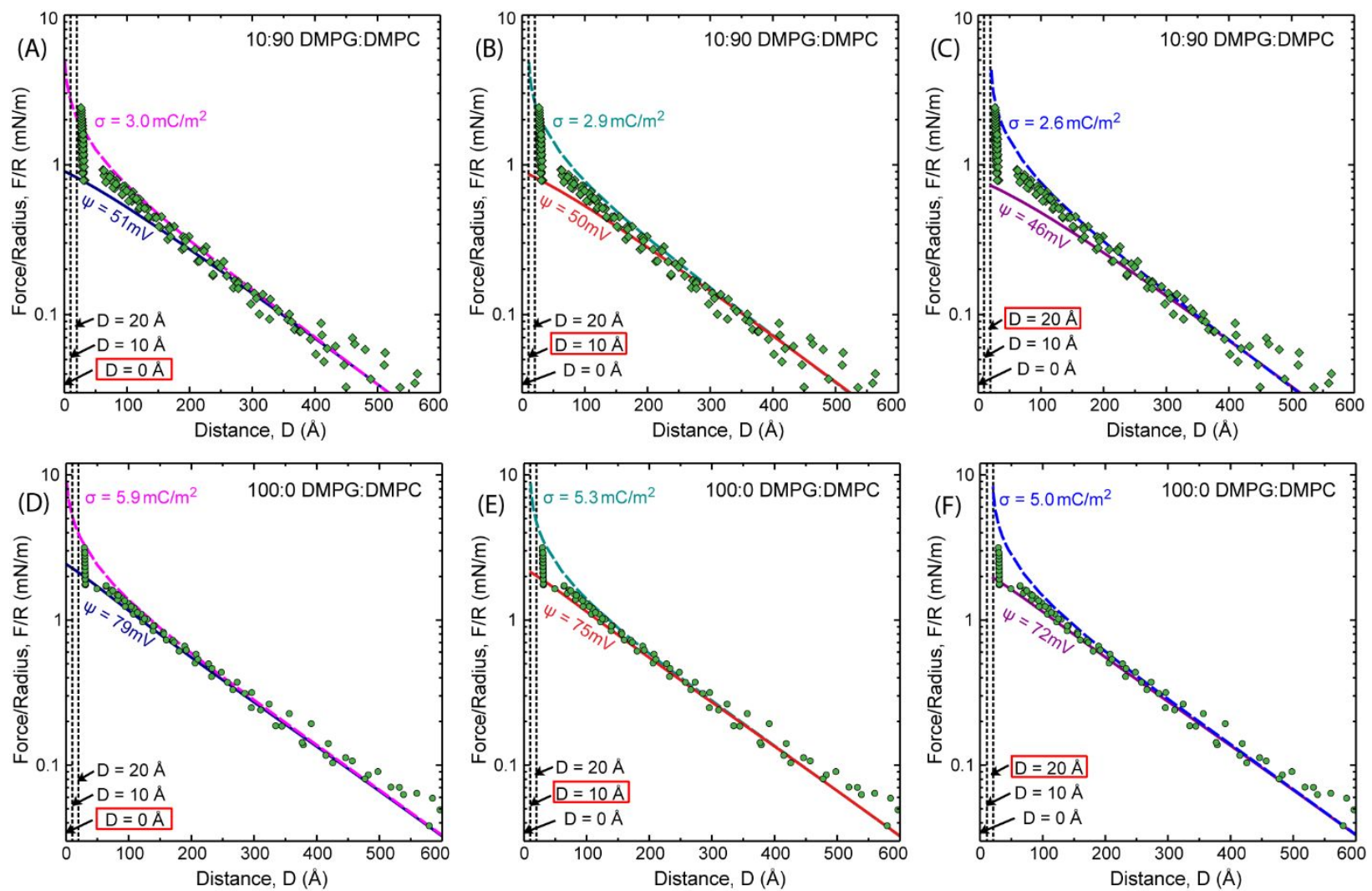

Figure S5: Measured force/radius vs distance profile between lipid bilayers composed of 10:90 DMPG:DMPC (A-C), and 100:0 DMPG:DMPC (D-F) deposited on DPPE (inner leaflet) in $0.5 \mathrm{mM} \mathrm{NaNO}_{3}\left(\mathrm{pH} \mathrm{5.7,25.0}{ }^{\circ} \mathrm{C}\right)$ on a semi-logarithmic plot. The assumption for plane of origin of charge is varied between $\mathrm{D}=0,10$ and $20 \AA$ (as indicated on the graph) and the surface charge and potential were fit using the non-linear Poisson Boltzmann equation.

The soft, fluid nature of lipid bilayers complicates an exact/absolute definition of charge origin due to membrane undulations, the ability of water molecules and counterions to penetrate and adsorb at the membrane interface, and capacity for lipid membrane reorganization. Through this analysis we have covered the most relevant/possible planes of charge origin. $\mathrm{D}=0 \AA$ corresponds to contact between the anhydrous lipid bilayers (without hydration) and is the approximate location of the phosphate head group plane that bears ionizable moieties. A surface separation of $\mathrm{D}=10 \AA$ (5 $\AA$ away from each surface) corresponds to contact between the two bilayer stern layers (the location of the outer Helmholtz plane from which the diffuse layer originates). $\mathrm{D}=20 \AA$ 
corresponds approximately with the hydrated bilayer contact around which hydration forces begin to dominate over electrostatic repulsion forces.

Figure S5 shows the range of charge and potential values obtained when the three aforementioned charge planes of origin are used for fitting/interpreting electrostatic forces measured between lipid bilayers using the SFA. The electrostatic forces (or energies) predicted by solutions of the non-linear Poisson Boltzmann equation based on constant charge or constant potential boundary conditions are in good agreement with each other at large surface separations (greater than $200-300 \AA$ ) but start to deviate at smaller surface separations. The experimentally measured forces lie between the two fits indicating that the surfaces exhibit charge regulation behavior. ${ }^{2}$ Different assumptions result in a deviation of $\pm 0.5 \mathrm{mC} / \mathrm{m}^{2}$ in charge or $\pm 5 \mathrm{mV}$ in potential which is comparable to the standard deviation of the experiments. At low salt conditions ( $0.5 \mathrm{mM}$ salt, $\mathrm{\kappa}^{-1} \sim 135 \AA$ ), the different charge plane of origin do not result in significant differences in surface potential values and are consistent with zeta potential measurements.

1. Skaug, M. J.; Longo, M. L.; Faller, R., The impact of Texas Red on lipid bilayer properties. The Journal of Physical Chemistry B 2011, 115 (26), 8500-8505.

2. Grabbe, A., Double layer interactions between silylated silica surfaces. Langmuir 1993, 9 (3), 797-801. 